\title{
The neon abundance in WC stars. II. ISO-SWS spectroscopy of WR 90 (HD 156385)
}

\author{
Luc Dessart, Allan J. Willis and Paul A. Crowther \\ Department of Physics \& Astronomy, UCL, Gower St., London, \\ WC1E $6 B T, U K$
}

Patrick W. Morris

SRON, Sorbonnelaan 2, NL-3584 CA, Utrecht, the Netherlands

D. John Hillier

Department of Physics 8 Astronomy, University of Pittsburgh, Pittsburgh, PA 15260, USA

\section{Introduction}

In general, observationally derived wind compositions of WR stars are in reasonable agreement with predictions from stellar evolution models for massive stars. However, Barlow et al. (1988) identified a major discrepancy for neon in $\gamma^{2}$ Vel (WC8+O) using ground-based observations. The advent of the ESA Infrared Space Observatory (ISO) has allowed the study of neon in many more WC stars, using mid-IR fine structure lines ([NeII] $12.81 \mu \mathrm{m}$, [NeIII] $15.55 \mu \mathrm{m}$ and $[\mathrm{Nev}] 14.32 \mu \mathrm{m})$. Willis et al. (1998) used ISO-sWs observations of WR 146 (WC5+O) to derive a neon abundance that was within the range expected theoretically. Here we undertake a study of WR 90 (HD 156385), the only (apparently) single WC7 star in our Galaxy, using ISO-sws spectroscopy. The only spectroscopic neon feature in the mid-IR of WR 90 is [NeIII] $15.55 \mu \mathrm{m}$, in addition to numerous $\mathrm{C}$ IV and He II transitions (see Figure 1).

\section{Stellar and chemical properties of WR 90}

In order to derive the neon abundance of WR 90, its stellar parameters need to be determined. These were obtained using the Hillier $(1989,1990)$ and Hillier \& Miller (1998) non-LTE atmospheric models for WR winds, the latter allowing for clumping and line blanketing. As pointed out by Willis et al. (1998), the neon-abundance determination is highly dependent on the mass-loss rate, which is sensitive to clumping in the wind. We found that a strongly clumped wind was necessary to reproduce the line-profile electron-scattering wings, so that the preferred mass-loss rate was substantially lower than the homogeneous case. The ISO-sws observations, together with UV (IUE) and optical (AAT) flux calibrated data sets allowed the mass-loss rate and interstellar extinction $\left(E_{B-V}=0.4 \mathrm{mag}\right)$ of WR 90 to be tightly constrained. For our assumed $M_{v}=-4.7 \mathrm{mag}$ (obtained from cluster WC7 stars), we obtain a distance to WR 90 of $1.4 \mathrm{kpc}$. The majority of spectral lines over the entire wavelength range 

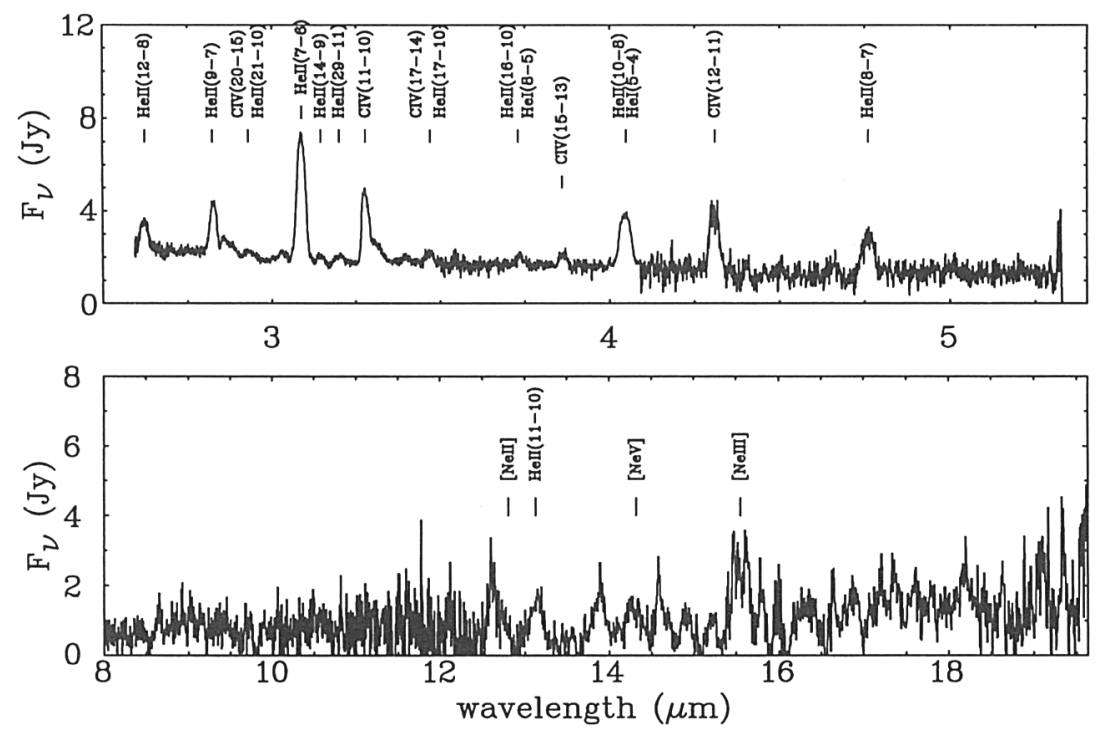

Figure 1. Flux-calibrated spectrum of WR 90 (HD 156385, WC7) obtained with ISO-sws, including the [NeIII] $15.55 \mu \mathrm{m}$ feature.

were well reproduced with the following parameters: $\dot{M}=2.5 \times 10^{-5} \mathrm{M}_{\odot} \mathrm{yr}^{-1}$, $\log L / \mathrm{L}_{\odot}=5.5, R_{*} \simeq 3.5 \mathrm{R}_{\odot}, v_{\infty}=2050 \mathrm{~km} \mathrm{~s}^{-1}$ and $\mathrm{C} / \mathrm{He}=0.35$, by number. Line-blanketing led to an increase in the derived stellar temperature and luminosity, with a lower carbon abundance and mass-loss rate obtained.

Following the technique of Barlow et al. (1988), we derived the abundance of $\mathrm{Ne}^{2+}$. Since Ne IV does not have any fine structure line in its ground state, we deduce a upper bound for the neon abundance by assuming $\mathrm{Ne}^{3+} \ll \mathrm{Ne}^{2+}$. We finally obtain a lower limit of $\mathrm{Ne} / \mathrm{He} \geq 8 \times 10^{-3}$, by number, in good agreement with expectations from evolutionary calculations. Similar detailed studies are being carried out for an additional five WC stars, observed with ISO-sWs.

Acknowledgments. This work is based on observations with ISO, an ESA project with instruments funded by ESA Member States (especially the PI countries: France, Germany, the Netherlands and the UK) with the participation of ISAS and NASA. We thank Tim Harries for obtaining optical spectroscopy of WR 90 at the AAT.

\section{References}

Barlow, M.J., Roche, P., Aitken, D.A. 1988, MNRAS 232, 821

Hillier, D.J. 1989, ApJ 347, 392

Hillier, D.J. 1990, ApJ 231, 116

Hillier, D.J., Miller, D.L. 1998, ApJ 496, 407

Willis, A.J., Dessart, L., Crowther, P.A., Morris, P.W., Maeder, A.,Conti, P.S., van der Hucht, K.A. 1988, MNRAS 290, 371 